\title{
PRODUCTS OF REFLECTIONS IN THE UNITARY GROUP
}

\author{
DRAGOMIR Ž. DJOKOVIĆ AND JERRY MALZAN
}

\begin{abstract}
Let $A \in U(n), \operatorname{det}(A)= \pm 1$ and let $\exp \left(i \alpha_{k}\right), 1<k<n$ be the eigenvalues of $A$ where $0<\alpha_{1}<\alpha_{2}<\cdots<\alpha_{n}<2 \pi$. Then $k(A)=\left(\alpha_{1}\right.$ $\left.+\cdots+\alpha_{n}\right) / \pi$ is an integer and $0<k(A)<2 n-1$. Denote by $l(A)$ the length of $A$ with respect to the set of all reflections, i.e., $l(A)$ is the smallest integer $m$ such that $A$ is a product of $m$ reflections. A reflection is a matrix conjugate to $\operatorname{diag}(-1,1, \ldots, 1)$. Our main result is the formula $l(A)=$ $\max \left(k(A), k\left(A^{*}\right)\right)$.
\end{abstract}

Introduction. By a reflection we mean here an element $R$ of the unitary group $U(n)$ such that $R^{2}=I_{n}$ and $\operatorname{rank}\left(R-I_{n}\right)=1$. We consider $U(n)$ as operating on the space of column vectors $\mathbf{C}^{n}$.

The reflections in $U(n)$ generate a normal subgroup $G(n)$ which consists of all matrices $A \in U(n)$ with $\operatorname{det}(A)= \pm 1$. It was shown by $\mathrm{H}$. Radjavi [1] that in fact every element of $G(n)$ is a product of at most $2 n-1$ reflections and he conjectured that this bound is the best possible.

It is a simple consequence of our Theorem below that this conjecture is correct.

If $A=R_{1} R_{2} \cdots R_{m}$ where $R_{k}$ are reflections and $m$ is minimal, then we say that $m$ is the length of $A$ and we denote it by $l(A)$.

For $A \in U(n)$ there exist uniquely determined real numbers $\alpha_{1}, \ldots, \alpha_{n}$ satisfying $0<\alpha_{1} \leqslant \alpha_{2}<\cdots<\alpha_{n}<2 \pi$ and such that $A$ is conjugate to the diagonal matrix

$$
\left(\begin{array}{ccc}
e^{i \alpha_{1}} & & 0 \\
& \ddots & \\
0 & & e^{i \alpha_{n}}
\end{array}\right)
$$

We shall say that $\alpha_{1}, \ldots, \alpha_{n}$ are the angles of $A$. The sum of the angles plays a very important role and we shall write

$$
\alpha_{1}+\cdots+\alpha_{n}=k(A) \cdot \pi .
$$

Note that $k(A)$ is an integer if and only if $A \in G(n)$.

In this paper we shall prove the following result.

THEOREM. If $A \in G(n)$ then $l(A)=\max (k(A), k(\bar{A}))$.

Received by the editors April 4, 1978.

AMS (MOS) subject classifications (1970). Primary 20G20, 22C05; Secondary $22 E 15$.

Key words and phrases. Unitary group, reflections, length, eigenvalues, angles, characteristic polynomial.

(c) 1979 American Mathematical Society 0002-9939/79/0000-0053/\$02.00 
For instance, if the angles $\alpha_{1}, \ldots, \alpha_{n}$ of $A$ satisfy $0<\alpha_{k}<\pi(1 \leqslant k \leqslant n)$ and $\alpha_{1}+\cdots+\alpha_{n}=\pi$ then $k(A)=1, k(\bar{A})=2 n-1$ and consequently $l(A)=2 n-1$. Thus, Radjavi's conjecture is correct.

Proof of the Theorem. The proof will be given in a sequence of several lemmas.

If $a \in \mathbf{C}^{n}$ is a unit vector, i.e., $a^{*} a=1$, then we denote by $R_{a}$ the reflection $I_{n}-2 a a^{*}$. The hyperplane orthogonal to $a$ is fixed pointwise by $R_{a}$ and $R_{a}(a)=-a$.

LEMMA 1. Let $A \in U(n)$ be diagonal with eigenvalues $\lambda_{1}, \ldots, \lambda_{n}$. Then the characteristic polynomial $\phi(\lambda)$ of $R_{a} A$ is

$$
\phi(\lambda)=\left\{\prod_{k=1}^{n}\left(\lambda-\lambda_{k}\right)\right\} \cdot \sum_{k=1}^{n} \frac{\lambda+\lambda_{k}}{\lambda-\lambda_{k}}\left|a_{k}\right|^{2}
$$

where the $a_{k}$ are the coordinates of the unit vector $a$.

Proof. We have

$$
\begin{aligned}
\phi(\lambda) & =\operatorname{det}\left(\lambda I_{n}-R_{a} A\right)=-\operatorname{det}\left(\lambda R_{a}-A\right) \\
& =-\operatorname{det}\left(\lambda I_{n}-A-2 \lambda a a^{*}\right) \\
& =-\operatorname{det}\left(\lambda I_{n}-A\right) \cdot \operatorname{det}\left(I_{n}-2 \lambda\left(\lambda I_{n}-A\right)^{-1} a a^{*}\right) .
\end{aligned}
$$

Since $\left(\lambda I_{n}-A\right)^{-1} a a^{*}$ has rank 1 , its eigenvalues are 0 (with multiplicity $n-1)$, and $a^{*}\left(\lambda I_{n}-A\right)^{-1} a$. Therefore

$$
\phi(\lambda)=-\operatorname{det}\left(\lambda I_{n}-A\right) \cdot\left(1-2 \lambda a^{*}\left(\lambda I_{n}-A\right)^{-1} a\right) .
$$

Since $\left|a_{1}\right|^{2}+\cdots+\left|a_{n}\right|^{2}=1$, this is equivalent to the formula (2).

LEMMA 2. Let $A \in U(n)$ have the angles $\alpha_{1}, \ldots, \alpha_{n}$. If $R \in U(n)$ is a reflection and if $\beta_{1}, \ldots, \beta_{n}$ are the angles of $R A$, then

$$
\beta_{1}+\cdots+\beta_{n}=\alpha_{1}+\cdots+\alpha_{n} \pm \pi \text {. }
$$

Proof. Let $\lambda_{1}, \ldots, \lambda_{m}$ be the distinct eigenvalues of $A$. Let $V_{k}$ be the $\lambda_{k}$-eigenspace of $A$. Finally, let $H$ be the hyperplane of $R$-fixed vectors and $W_{k}=V_{k} \cap H$. It is clear that $\operatorname{dim}\left(V_{k} / W_{k}\right)=0$ or 1 for each $k$. The subspace $W=W_{1}+\cdots+W_{m}$ is invariant under both $R$ and $A$. Hence $L=W^{\perp}$ is also invariant under $R$ and $A$. Let $R_{0}$ and $A_{0}$ be their respective restrictions to $L$. It is clear that all eigenvalues of $A_{0}$ are simple and that it suffices to prove the assertion for $R_{0}$ and $A_{0}$ instead of $R$ and $A$. In other words we may assume now that $A$ has only simple eigenvalues.

Without loss of generality we may assume that $A$ is given by (1). Let $a$ be a unit vector such that $R=R_{a}=I_{n}-2 a a^{*}$. By conjugating both $A$ and $R$ by a suitable permutation matrix, we may assume that the first $m$ coordinates $a_{1}, \ldots, a_{m}$ of $a$ are nonzero while $a_{m+1}=\cdots=a_{n}=0$. Consequently $A$ and $R A$ have the angles $\alpha_{m+1}, \ldots, \alpha_{n}$ in common. Hence it suffices to prove the lemma in the case when $m=n$, i.e., when all $a_{k} \neq 0$.

Now let $\lambda_{k}=e^{i \alpha_{k}}, 1 \leqslant k<n$. By Lemma 1 , the characteristic polynomial 
$\phi(\lambda)$ of $R A$ is given by (2). Putting $\lambda=e^{i \theta}$ the equation $\phi(\lambda)=0$ becomes

$$
\sum_{k=1}^{n}\left|a_{k}\right|^{2} \operatorname{cotan} \frac{1}{2}\left(\theta-\alpha_{k}\right)=0 .
$$

Now we may assume that $0 \leqslant \alpha_{1}<\alpha_{2}<\cdots<\alpha_{n}<2 \pi$. It is immediate from (3) that there is at least one root of (3) in each of the intervals $\left(\alpha_{k}, \alpha_{k+1}\right)$ for $1<k<n-1$ and another one in $\left(\alpha_{n}, \alpha_{1}+2 \pi\right)$. Hence the angles $\beta_{1}, \ldots, \beta_{n}$ of $R A$ ordered so that $0<\beta_{1}<\beta_{2} \leqslant \cdots<\beta_{n}<2 \pi$ will satisfy either

$$
0<\beta_{1}<\alpha_{1}<\beta_{2}<\alpha_{2}<\cdots<\beta_{n}<\alpha_{n}
$$

or

$$
\alpha_{1}<\beta_{1}<\alpha_{2}<\cdots<\beta_{n-1}>\alpha_{n}<\beta_{n}<2 \pi .
$$

In case (4) we have

$$
\alpha_{1}+\cdots+\alpha_{n}>\beta_{1}+\cdots+\beta_{n}<\alpha_{1}+\cdots+\alpha_{n}-2 \pi
$$

and in case (5) we have

$$
\alpha_{1}+\cdots+\alpha_{n}<\beta_{1}+\cdots+\beta_{n}<\alpha_{1}+\cdots+\alpha_{n}+2 \pi \text {. }
$$

Since $\operatorname{det}(R A)=-\operatorname{det} A$ the difference

$$
\left(\beta_{1}+\cdots+\beta_{n}\right)-\left(\alpha_{1}+\cdots+\alpha_{n}\right)
$$

must be an odd multiple of $\pi$ and the proof is completed.

For $A \in G(n)$ let $k^{\prime}(A)=\max (k(A), k(\bar{A}))$.

LEMMA 3. If $A \in G(n)$ and $R$ is a reflection then $k^{\prime}(R A)=k^{\prime}(A) \pm 1$. Consequently, $l(A) \geqslant k^{\prime}(A)$.

Proof. Let $r=\operatorname{rank}\left(A-I_{n}\right)$ and let $\alpha_{1}, \ldots, \alpha_{r}$ be the nonzero angles of $A$. Then the nonzero angles of $\bar{A}$ are $2 \pi-\alpha_{1}, \ldots, 2 \pi-\alpha_{r}$. Thus $k(A)+$ $k(\bar{A})=2 r$, and so the difference $k(A)-k(\bar{A})$ is even. Therefore it suffices to prove that $k(R A)=k(A) \pm 1$. But this last claim is immediate from Lemma 2.

LEMma 4. Let $\alpha, \beta$ be two angles of $A \in U(n)$ such that $0<\alpha<\pi<\beta<$ $2 \pi$. Then there exists a reflection $R$ such that the angles of $R A$ are the same as those of $A$ except that $\alpha$ and $\beta$ are replaced by 0 and $\alpha+\beta-\pi$.

Proof. Clearly, it suffices to consider the case when $n=2$. We need only to show that we can choose a reflection $R=R_{a}$ so that $R A$ has 1 as eigenvalue. The equation (3) will be satisfied by $\theta=0$ if and only if

$$
\left|a_{1}\right|^{2} \operatorname{cotan} \frac{\alpha}{2}+\left|a_{2}\right|^{2} \operatorname{cotan} \frac{\beta}{2}=0 \text {. }
$$

One can choose $a_{1}$ and $a_{2}$ nonzero satisfying this equation because the two cotangents have opposite signs.

LEMMA 5. If $A \in G(n)$ and $k^{\prime}(A) \geqslant 1$, there exists a reflection $R$ such that $k^{\prime}(R A)=k^{\prime}(A)-1$. 
Proof. We may assume that $A$ is given by (1) where $\alpha_{1}, \ldots, \alpha_{n}$ are the angles of $A$ in increasing order. If some $\alpha_{k}=\pi$ it suffices to take $R=I_{n}-$ $2 e_{k} e_{k}^{*}, e_{k}$ being the standard basic vector of $\mathbf{C}^{n}$.

If $\alpha_{n}<\pi$ we can take $R=I_{n}-2 e_{n} e_{n}^{*}$.

If $\alpha_{1}>\pi$ we can take $R=I_{n}-2 e_{1} e_{1}^{*}$.

Finally, if $0<\alpha_{k}<\pi<\alpha_{r}<2 \pi$ for some $k, r$ then we can apply Lemma 4.

The assertion of the Theorem follows from Lemmas 3 and 5.

\section{REFERENCES}

1. H. Radjavi, Decomposition of matrices into simple involutions, Linear Algebra and Appl. 12 (1975), 247-255.

Department of Pure mathematics, University of Waterloo, Waterloo, Ontario, CaNADA N2L 3G1 (current address of D. Ż. Djoković)

Current address (Jerry Malzan): Department of Mathematics, University of Toronto, Toronto, Ontario, Canada M5S 1A1 